\title{
A Novel Optical Coherence Tomography for Non-Destructive Characterisation of Electrical-Thermal-Mechanical Deformation of Bonding Wires
}

\author{
Zhiyi Zhao, Zijian Zhang and Yaochun Shen \\ (ycshen@liverpool.ac.uk) \\ Department of Electrical Engineering and Electronics, The University of Liverpool, \\ Liverpool L69 3GJ, UK
}

\begin{abstract}
Bonding wire is one of the most failure-prone components of power electronics modules, and silicone gel is usually employed to encase bonding wires. In this talk I will report the use of optical coherence tomography (OCT) technique to precisely measure the electrical-thermal-mechanical (ETM) deformation of bonding wires under silicone gel. A spectraldomain line-field OCT system was developed to capture the whole cross-sectional image of the bonding wire sample in a single-shot fashion without the need of any mechanical scanning. The time-resolved displacement/deformation of the bonding wire under silicone gel can be measured with sub-nanometre sensitivity by extracting the phase information recorded in the raw OCT spectrogram. The achieved temporal resolution is $2.5 \mathrm{~ms}$ for monitoring the ETM deformation dynamics of the bonding wire. We found that the ETM deformation of the gel-encased bonding wire was about three times smaller than that of the bare bonding wire. These experimental results suggest that the spectral-domain line-field OCT could be a useful analytical tool for studying the time-dependent ETM deformation of bonding wires encased by silicone gel.
\end{abstract}

\section{Reference}

[1] Z. Zhao, Z. Zhang, S. Lawman, Z. Yin, Y. Hu, J. Xu, and Y.C. Shen, Characterisation of Electrical-Thermal-Mechanical Deformation of Bonding Wires Under Silicone Gel Using LF-OCT, IEEE Transactions on Power Electronics, (http://doi.org/10.1109/TPEL.2021.3068128) 